\title{
FROM BIM MODELS TO INTEGRATED ENERGY EFFICIENCY APPLICATIONS FOR INDUSTRIAL FACILITIES
}

\author{
Georgios Gourlis ${ }^{1}$, Peter Smolek ${ }^{2}$, Bernhard Heinzl ${ }^{3}$, Ines Leobner ${ }^{4}$ and \\ Iva Kovacic ${ }^{5}$
}

\begin{abstract}
Raised interest in efficient use of available resources in industrial facilities prompts for software-tools to predict and manage energy demand of the whole system. An integrated approach considering all energy consumption contributors, classified into building, energy system, production and logistics, is developed within the research project Balanced Manufacturing (BaMa). Thereby BIM models of industrial spaces containing valuable information are used for creating a comprehensive representation of building-related aspects in a hybrid simulation environment, able to assess manufacturing and auxiliary energy demands.

For testing the applicability of this integrated hybrid approach, a prototype is developed based on an actual use case. The simplification procedure is presented and the energy performance results are compared with that of an established building energy modelling software, discussing advantages and limitations. Essential information that needs to be transferred from the BIM model is clarified and prioritised.
\end{abstract}

Keywords: energy efficient production, building energy modelling, waste heat emissions, hybrid simulation.

\section{INTRODUCTION}

The industrial sector is responsible for $26 \%$ of the energy consumption in Europe (Eurostat 2016). With the implementation of the EU Energy Efficiency Directive and the recently proposed strengthening of the set targets (European Commission), it is becoming increasingly important for industrial facilities to efficiently use available resources and identify significant potential savings, having both economic and ecological impact. Interdisciplinary simulation-based approaches are proposed to size industrial energy consumption and optimise its efficiency (e.g., Herrmann et al. 2011; Bleicher et al. 2014). This requires modelling of complex systems, with both continuous and discrete aspects, to balance performance and interaction of elements such as the building envelope, technical building services (TBS) and manufacturing processes.

1 Research Assist. Dipl.-Ing. MSc, Department of Industrial Building and Interdisciplinary Planning, Faculty of Civil Engineering, TU Wien, Austria, georgios.gourlis@tuwien.ac.at

2 Research Assist. Dipl.-Ing, Institute for Energy Systems and Thermodynamics, Faculty of Mechanical and Industrial Engineering, TU Wien, Austria, peter.smolek@tuwien.ac.at

3 Research Assist. Dipl.-Ing., Institute of Computer Aided Automation, Faculty of Informatics, TU Wien, Austria, bernhard.heinzl@tuwien.ac.at

4 Research Assist. Dipl.-Ing. Dr.techn., Institute for Energy Systems and Thermodynamics, Faculty of Mechanical and Industrial Engineering, TU Wien, Austria, ines.leobner@tuwien.ac.at

5 Associate Prof. Dipl.-Ing. Dr.techn., Department of Industrial Building and Interdisciplinary Planning, Faculty of Civil Engineering, TU Wien, Austria, iva.kovacic@tuwien.ac.at 
In this context, Building Information Modelling (BIM), defined as "a digital representation of physical and functional characteristics of a facility" (buildingSMARTalliance 2007), offers potentials through creation of a joint knowledge database for follow up analysis of buildings and building systems in terms of energy performance. Still, data rich BIM models need to be simplified to provide other specific tools only certain information, essential for the accomplishment of the analysis. Extensive research has been conducted on data transfer from BIM to Building Energy Modelling (BEM) tools (Rose and Bazjanac 2015). However, such a process is yet far from standardised in the general practise as ready for analysis realisable models cannot be generated from initial architectural BIM models, which are not designed for that purpose (Gourlis and Kovacic 2017). Even with an appropriate BEM model, it is rather challenging for building performance simulation software to incorporate manufacturing related internal heat gains with sufficient level of detail, which affect indoor climate conditions and can significantly vary based on production cycles.

In order to address the need of an integrated approach to energy and resource efficient operation of industrial facilities, the research project Balanced Manufacturing (BaMa) ${ }^{6}$ was initiated. The aim is to develop a simulation-based toolchain that integrates energy demand and carbon emissions as control parameters in industrial plant operation. Through monitoring, predicting and optimising the energy and resource demand of the production as a whole, as well as that of individual products, BaMa intends to provide performance indicators and management of a facility's energy demand.

In this paper, we present the information flow from a BIM model of an industrial production unit to the first prototype of the integrated hybrid simulation model of the BaMa toolchain. Focus is given to building related aspects and the necessary abstraction procedure for dismantling the facility to basic components in order to build a simple bottom-up test model. A performance comparison of energy demand between an EnergyPlus BEM model and the hybrid simulation model shows potentials and deficits of each approach. We prioritise then the BIM related information that is needed for proceeding to holistic assessment of a facility's energy demand.

\section{BACKGROUND}

In the course of the BaMa project, a generic methodology was formulated by dividing the overall system into well-defined manageable modules called "cubes", enabling a focused analysis of highly complex and heterogeneous systems in a variety of production facilities. The cubes represent physical parts of the facility and are mapped into mathematically formulated virtual counterparts in a simulation model. They are defined and connected through common interfaces, combining three kinds of flows: energy, material (incorporating the immediate value stream of products) and information (control and demand related). Energy flows and related carbon emissions are described by continuous values, whereas material and product flows have to be described as discrete entities. Combining these aspects requires hybrid simulation capabilities. Some approaches for simulating hybrid systems employ co-simulation by coupling different simulation environments via middleware. For example, the BCVTB (Building Controls Virtual Test Bed) described by Wetter (2011) provides coupling of several software tools common for building performance simulation, like EnergyPlus, Dymola/Modelica, MATLAB and others. While this type of simulation allows employing familiar simulation environments

6 http://bama.ift.tuwien.ac.at 
and model descriptions (see also Heinzl et al. 2014), it typically introduces significant complexity into the modelling process, in particular regarding model development, maintainability and reusability of developed components. In the scope of BaMa, Modelica and EnergyPlus also lack functionality for simulating event-driven models of discrete production entities.

As a consequence, we opted for an integrated hybrid approach for the BaMa toolchain that allows describing self-contained, loosely coupled and reusable hybrid model components in a single simulation environment. This approach is based on the DEV\&DESS (Discrete Event and Differential Equation System Specification) formalism as described by Zeigler et al. (2000).

The comprehensive BaMa approach takes into account four fields that constitute an industrial facility addressing the building itself, the TBS, the production processes and the logistics. Especially for the building aspect, the aim of the BaMa toolchain is to deliver information about auxiliary energy demand, such as space heating and cooling, based on weather conditions and production schedules. Together with production needs, the toolchain will be able to predict the energy demand of the entire facility, when provided with production schedules and weather forecasts. Consequently this enables optimisation of the systems' operation via algorithms, by using parameters as energy saving, costs reduction or time as target function. An extensive description of the BaMa methodology is available in Leobner et al. (2015).

\section{MODELS}

First the essential cube models from which an industrial facility is composed are briefly discussed. Further on, this section presents the simplification process from a pre-modelled architectural BIM model to the first prototype of the BaMa hybrid simulation model on the case study of a food industry building, consisting of two blocks - a bakery and meat factory. Focus is the bakery block, which produces baked goods, both fresh and frozen.

\subsection{The cube model}

According to the "cubes" approach, the building consists of a building cube and thermal zone cubes. The first represents the solid constructions, i.e. walls, slabs, roof etc., and also calculates the heat exchange between neighbouring thermal zones and to the outside. The second describes the thermal condition of a space, calculating the heating and cooling demand. For that purpose it is necessary to assign the adjacent surfaces between the zones through a mapping process.

The current cube model considers heat transfer between thermal zones and the surrounding only by heat conduction. Other effects such as the influence of solar radiation, shading and the wind velocity can be incorporated into the cube model structure; however, this is still work in progress. Windows are also considered in regards to the thermal conductivity only.

The energy systems are described by the energy converter and energy grid cubes. The first convert energy from one type to another, a process that may be accompanied by losses emitted for example as waste heat to the surrounding thermal zone. The grid distributes energy from one or more sources to consumers (e.g., machines, ventilation, etc.) providing an additional energy storage capacity if needed. Building and energy related cubes have only continuous behaviour. Production and logistics cubes can additionally be entered and exited by discrete entities, e.g., products, triggering a change in their continuous behaviour. 
As a detailed presentation of the cube models would go beyond the scope of this paper, discursive information can be found in the publications by Smolek et al. (2016) and Raich et al. (2016).

\subsection{First stage simplification}

For the purpose of applying the BaMa methodology for studying the energy performance of the facility an appropriate definition of thermal zones, based on space usage in relation to production stages, construction properties and space conditioning requirements takes place. This constitutes the first stage simplification of the information contained in the Revit BIM model to form an EnergyPlus thermal simulation. As the original BIM model was not planned to be used for such a purpose, it required extensive rework. The initial model contained room-stamps definitions according to the architectural plans being too detailed for the BEM model. Therefore from a number of 113 room-stamps, the BEM model resulted in only 22 thermal zones after the abstraction, redefining internal boundaries and applying the appropriate material characteristics of the bounding constructions. Geometry information of the external building envelope remained intact. The model was transferred from Revit to EnergyPlus via the OpenStudio plug-in for SketchUp, a detailed presentation of the workflow method for the transfer as well as identified problems and applied solutions can be found in Gourlis and Kovacic (2016). Material thermal properties were directly applied on the EnergyPlus model, as it was not possible to export them from BIM via the gbXML file format. Information lost in the process was the surface areas of interior walls inside single zones as well as piping and ducts of TBS, which together would contribute to the internal mass of the thermal zones.

\subsection{Second stage simplification}

Even if the building was already simplified, the representation of the whole production cycle and accompanying logistics as well as the energy systems was quite challenging, hence it was advisable to construct a simple model prototype to test the outcome of the hybrid simulation (Figure 1), for which we employed the cube approach. First the facility was scaled down to a new model geometry that contains the main functions of production spaces and necessary storage, technical and administrative areas of the previous model and omits secondary spaces, while maintaining thermal zone spatial adjacencies and volume characteristics. The abstraction process reduced the number of zones to only four elemental areas for the operation of the hypothetical facility, keeping though the variety of constructions of the different boundary surfaces and conditions (e.g., exterior and interior walls, walls to cold storage room). Space thermal condition requirements were also maintained. The simpler geometry of the prototype facilitated the mapping process of adjacent surfaces in the cubes model, however this model cannot be used at this stage to evaluate the performance of the original building.

The new simplified model contains a production hall, zone 1, with a double height ceiling that should sustain an indoor air temperature of $18^{\circ} \mathrm{C}$ to $28^{\circ} \mathrm{C}$ throughout the year. Zone 2 is housing the TBS and is not conditioned. Zone 3 is the cold storage area with a constant temperature at $4^{\circ} \mathrm{C}$. Zone 4 on the upper floor is the administration offices with temperatures ranging from $22^{\circ} \mathrm{C}$ to $26^{\circ} \mathrm{C}$. Furthermore, a production line able to deliver different products is modelled by production and logistic cubes. Respective ingredients in the form of simulation entities are pulled from storage and handed through mixing, splitting, and packaging stations as well as conveyor belts. The products can either pass an oven for baking or a freezer for cooling, depending on the type of product. Each station is supplied with electric energy, oven and freezer additionally receive thermal energy from 
respective energy grids. The energy system consists of an electric grid, a compression chiller (energy converter cube), a cold grid with storage capacity distributing cold to zones 1,3 and 4 and to the freezer and a gas heater (energy converter) with a heating grid serving zones 1, 3 and the oven. The cubes model of the prototype with entities and energy flows is shown in Figure 2.

Compared to common methods for incorporating production models into building energy simulation, like coupling EnergyPlus with Modelica (see also chapter 2), the BaMa cube approach provides an integrated description of all aspects in a single simulation environment, which facilitates reusability of model components, maintainability of the overall model as well as extendibility.
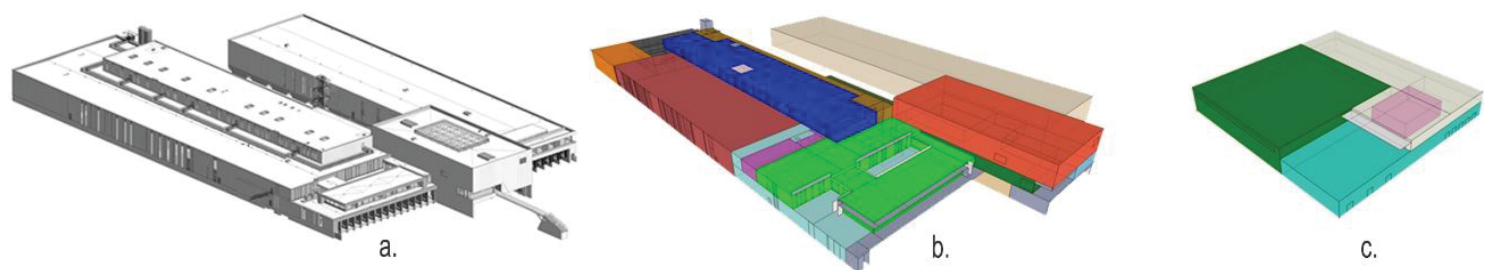

Figure 1: Two stage simplification process from the original BIM model (a), to EnergyPlus BEM model (b) and finally to the simple test prototype (c).

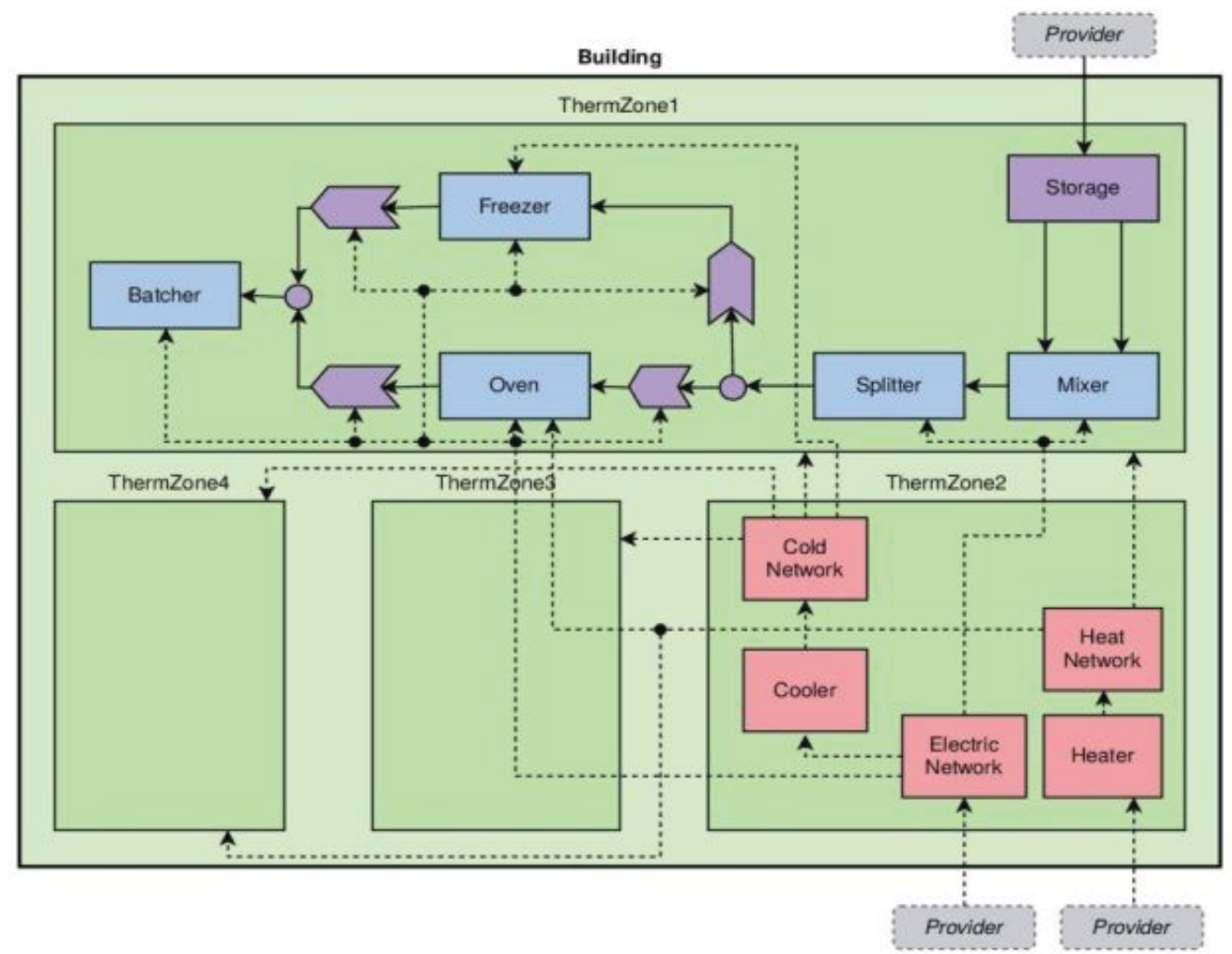

Figure 2: Prototype cubes model containing production machines, logistics components, energy systems and thermal zones. Dashed lines depict energy flows and solid lines material flows.

\subsection{Performance comparison}

To evaluate the energy performance of the test prototype implemented in the BaMa hybrid simulation environment in a specific simulation scenario, a BEM model of the building is 
also realised in the EnergyPlus software. Both models are given a production schedule for a typical day from 00:00 to 18:00, however, there is principle difference. On the one hand, BaMa hybrid model follows a detailed plan of products to be manufactured with appropriate machine hours for each product type, defining so the state of production and logistic cubes (setup, working and standby). On the other hand, EnergyPlus model is only given a constant average manufacturing loads value according to equipment wattage and the operation hours of the production line according to typical usage. This is expected to have an impact on the waste heat emissions from machinery, thus internal heat gains of the thermal zone. Simulations are then carried out over a course of one day in winter and in summer respectively. Table 1 presents the characteristics of each simulation model.

Table 1: Characteristics of the simulation models

\begin{tabular}{ccc}
\hline & BaMa hybrid & EnergyPlus \\
\hline Heat transfer calculation & conduction & conduction, convection, radiant \\
Zone temperature & homogenous-no air motion & homogenous-no air motion \\
Windows & only thermal conductivity - & fully considered - \\
opaque elements & transparent elements \\
Solar gains & not considered & direct and indirect \\
Production schedule & fully considered & not considered \\
Waste heat internal & dynamic & static - average \\
Energy system & covering requested demand & covering requested demand \\
Production process & detailed simulation of events & not available \\
Lighting / Ventilation & not considered & not considered
\end{tabular}

\section{RESULTS AND DISCUSSION}

\subsection{Simulation of production process}

Figure 3 shows the rate of internal heat gains throughout the day and the cumulative heating energy that is emitted in the thermal zone due to production processes. The BaMa hybrid model provides a dynamic progression of waste heat that would closer represent reality in a production facility. In this example, it predicts higher heat gains than the static approach of average loads, minimizing uncertainties included in the assumptive averages. The graphs also depict that production machines are shut down at 18:00 as planned. However, the outcome of the discrete event simulation of the hybrid model showed that all entities were already finished by 16:00, which would allow turning off the production line two hours earlier, thus saving energy.

\subsection{Energy performance}

Energy demand of the test prototype facility for the winter and summer scenario is shown in Figure 4. There are notable deviations between the two models originating from the different simulation models. As stated earlier, the BaMa model at this point does not consider transparent window surfaces. Given the fact that for both days there was increased direct solar radiation that led to high solar heat gains, in winter this led to lower 
heating demand and in summer to higher cooling for the EnergyPlus model. Another point is that gas heating in the EnergyPlus model is only used for space conditioning, whereas in the BaMa hybrid model the oven is also a consumer. The effects of the different system boundaries can be clearly seen in summer, where BaMa predicts a heating demand when there is no need for space heating. Production-oriented energy demand of the oven and the freezer is therefore contributing to the heating and cooling deviations.

Even for this test facility with a simple production line, the integrated simulation using the cubes approach allows the identification of dynamic interactions, e.g., the oven operation and the heating grid. Hence potential synergies across the whole system can be exploited in favour for decreasing the overall energy demand. However, the incorporation of transparent window surfaces in the hybrid simulation is vital for enabling valid predictions in actual industrial spaces. The achievement of a fully functional discrete event simulation along with a continuous energy assessment within a single integrated simulation environment can unveil additional energy saving measures. Calculation time efficiency of the BaMa approach over EnergyPlus is also documented.
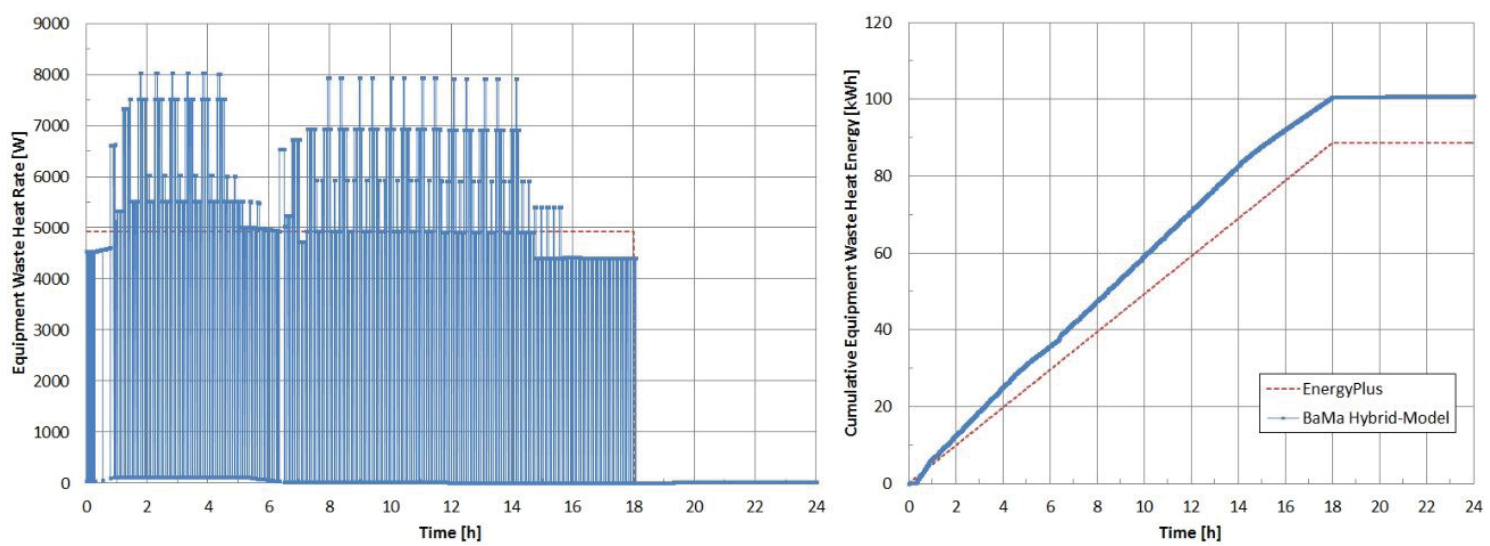

Figure 3: Production equipment waste heat rate and cumulative waste heat energy for the given production schedule.
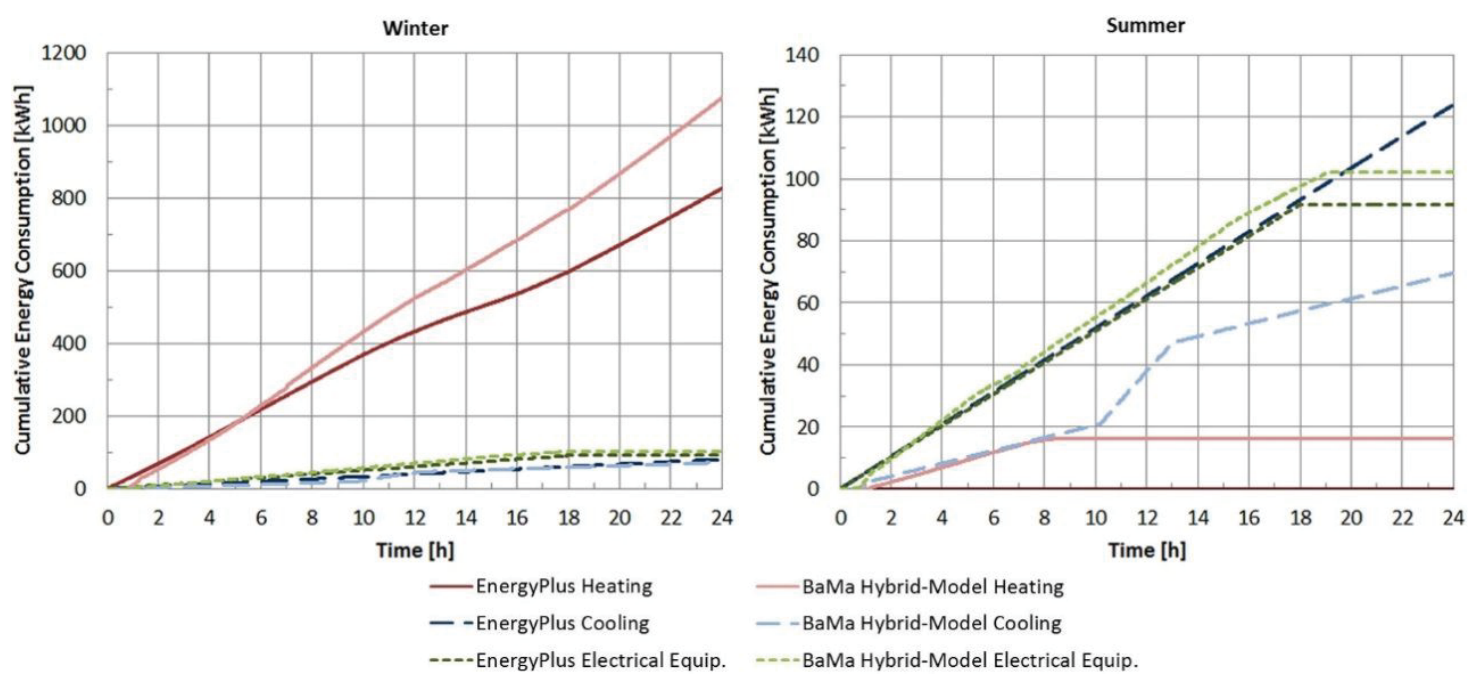

- BaMa Hybrid-Model Heatin

- -BaMa Hybrid-Model Cooling

--BaMa Hybrid-Model Electrical Equip.

Figure 4: Energy demand in winter and summer scenario.

\subsection{Essential building related information flow}

The exploratory testing of the prototype for the implementation of the BaMa simulation tool clarified the essential data that need to be delivered for constructing the virtual 
building cube. A BIM model could therefore export data sheets that would facilitate the input process in BaMa. First priority information is thermal zone function, area and volume as well as U-values and thermal properties of the zone boundary constructions, along with a type classification, e.g., exterior wall, floor, ceiling. Exterior surface area including appropriate orientation should be also assigned to each thermal zone. Crucial is the definition of interior zone separations by the adjacent surface area bearing particular attributes if these are in contact with special room conditions (e.g., refrigerator room).

Furthermore, on a second level, if available in the BIM model, internal gains sources as lighting, number of planned occupants and fixed electrical equipment can also be ascribed to zone characteristics. Lastly, masses of interior partitions, ducts and piping surfaces and, if designed in the BIM model, massive furnishing (e.g., high-rack storage system, tanks, machinery) within the set zones can be also be extracted. This will enhance the thermal storage capacity of the simulation model, as industrial halls are usually vast spaces, appearing empty in architectural plans but functioning at a fully-equipped level.

\section{CONCLUSIONS}

Industrial building BIM models can serve as valuable knowledge databases for energy efficiency tools as the one developed in the BaMa project. The test of a simplified prototype facility modelled in a hybrid simulation environment and its comparison to an established BEM software demonstrated potentials and deficits. Essential is the definition of the building thermal zones in BIM and the attribution of space, material, surface topology and energy loads information to them. The information flow from BIM to BaMa is currently a manual procedure, however, an automated data export function would assist the comprehensive representation on the building in the integrated hybrid model simulation environment. Open remains, if such a process is applicable on a detailed architectural BIM model through filtering options or if it would require a simpler model version of the building.

The incorporation of missing features and the expansion of the BaMa hybrid model application to the real case study of the bakery facility will further test its performance and usability through its validation against measurement data. Its implementation into the existing automation systems will not only provide a detailed energy management of entire facility but also indicate optimisation potentials of the overall performance.

\section{ACKNOWLEDGMENTS}

This research is a part of the Balanced Manufacturing (BaMa) project, funded by the Austrian Climate and Energy Funds within the program e!MISSION.at - Energy Mission Austria, grant number 840746 . We gratefully acknowledge the contribution of all partners.

\section{REFERENCES}

Bleicher, F., Duer, F., Leobner, I., Kovacic, I., Heinzl, B., and Kastner, W. (2014). Co-simulation environment for optimizing energy efficiency in production systems. CIRP Ann. Manuf. Technol., 63(1), pp. 441-444. dx.doi.org/10.1016/j.cirp.2014.03.122.

BuildingSMARTalliance. (2007). United States National Building Information Modeling Standard Version 1 - Part 1: Overview, Principles and Methodologies. National Institute of Building Science, Washington, D.C., USA. 
European Commission. (n.d.) Energy Efficiency Directive. Available at: https://ec.europa.eu/energy/en/topics/energy-efficiency/energy-efficiency-directive [Accessed: 4 Dec. 2016]

Eurostat. (2016). Energy balance sheets - 2014 data - 2016 edition. Available at: http://ec.europa.eu/eurostat/web/energy/data/energy-balances

[Accessed: 4 Dec. 2016]

Gourlis, G., and Kovacic, I. (2017). Building Information Modelling for analysis of energy efficient industrial buildings-A case study. Renew. Sustain. Energy Rev., 68(2), pp. 953-963. dx.doi.org/10.1016/j.rser.2016.02.009.

Heinzl, B., Kastner, W., Leobner, I., Dür, F., Bleicher, F., and Kovacic, I. (2014). Using coupled simulation for planning of energy efficient production facilities. Work. Model. Simul. Cyber-Phys. Energy Syst. doi: 10.1109/MSCPES.2014.6842397

Herrmann, C., Thiede, S., Kara, S., and Hesselbach, J. (2011). Energy oriented simulation of manufacturing systems-Concept and application. CIRP Ann.-Manuf. Technol., 60(1), pp. 45-48. dx.doi.org/10.1016/j.cirp.2011.03.127.

Leobner, I., Smolek, P., Heinzl, B., Kovacic, I., Ponweiser, K., Mayrhofer, W., Kastner, W., and Duer, F. (2015). "Balanced Manufacturing - a Methodology for Energy Efficient Production Plant Operation" In: Proc. 10th Conference on Sustainable Development of Energy, Water and Environment Systems (SDEWES 2015), paper-nr. SDEWES2015.0268.

Raich, P., B. Heinzl, F. Preyser, and W. Kastner, (2016) "Modeling techniques for integrated simulation of industrial systems based on hybrid PDEVS" In: 2016 Workshop on Modeling and Simulation of Cyber-Physical Energy Systems (MSCPES).

Rose, C. M., and Bazjanac, V. (2015). An algorithm to generate space boundaries for building energy simulation. Engineering with Computers, 31(2), pp. 271-280. doi:10.1007/s00366-013-0347-5.

Smolek, P., Leobner, I., Gourlis, G., Mörzinger, B., Heinzl, B., and Ponweiser, K. (2016). "Hybrid Building Performance Simulation Models for Industrial Energy Efficiency Applications" In: Proc. 11th Conference on Sustainable Development of Energy, Water and Environment Systems (SDEWES 2016), paper-nr. SDEWES2016.0134.

Wetter, M. (2011). Co-simulation of building energy and control systems with the building controls virtual test bed. Journal of Building Performance simulation, 4(3), pp. 185-203.

Zeigler, B. P., Praehofer, H., and Kim, T. G. (2000). Theory of modeling and simulation: integrating discrete event and continuous complex dynamic systems. Academic press. 